\title{
Some properties of the normalized periodogram of a fractionally integrated separable spatial ARMA (FISSARMA) model
}

\begin{abstract}
In this article, we study the properties of the normalized periodogram of the Fractionally Integrated Separable Spatial ARMA (FISSARMA) models. In particular, we establish the asymptotic mean of the normalised periodogram and the asymptotic second-order moments of the normalised Fourier coefficients. We also establish the asymptotic distribution of the normalised periodogram. Some numerical results are also provided.
\end{abstract}

Keyword: Fractionally spatial ARIMA model; Long memory; Normalised periodogram; Separable; Two-dimensional periodogram. 